\title{
The Role of the Adaptive Immune System in Burn-Induced Heterotopic Ossification and Mesenchymal Cell Osteogenic Differentiation
}

KR supported by the Coller Society Research Fellowship, NIH, F32 1-F32-AR-068902-01, and American Society of Maxillofacial Surgery.

SA Supported by the Coller Society Research Fellowship, NIH Loan Repayment Program, NIH, F32AR06649901A1 and Plastic Surgery Foundation

SL Supported by the Howard Hughes Medical Institute (HHMI) Medical Fellows Program

BL Supported by funding from NIH/National Institute of General Medical Sciences Grant K08GM109105-0, Plastic Surgery Foundation National Endowment Award, the Association for Academic Surgery Roslyn Award, American Association for the Surgery of Trauma Research \& Education Foundation Scholarship, DOD:

W81XWH-14-DMRDP- CRMRP-NMSIRA and American Association of Plastic Surgery Research Fellowship. Some of the authors are employees of the United States Government. This work was prepared as part of their official duties. Title 17 U.S.C. $\$ 105$ provides that "Copyright protection under this title is not available for any work of the United States Government." Title 17 U.S.C \$101 defined a US Government work as a work prepared by a military service member or employees of the United States Government as part of that person's official duties. The opinions or assertions contained in this paper are the private views of the authors and are not to be construed as reflecting the views, policy or positions of the Department of the Navy, Department of Defense nor the United States Government. This work was partially supported by DOD work units W81XWH14-2-0010 and 602115HP.3720.001.A1014. 


\begin{abstract}
Purpose: Heterotopic ossification $(\mathrm{HO})$ is the pathologic process of bone formation in soft tissues. Although the exact etiology remains unknown, inflammation appears to catalyze disease progression. The goal of this study is to determine the impact of the adaptive immune system on $\mathrm{HO}$.

Methods: $\mathrm{HO}$ was induced in 8-week-old control C57BL/6 and immunocompromised Rag1tm1Mom (Rag1 KO) male mice deficient in B-/T-lymphocytes via combined Achilles tenotomy and burn injury. MicroCT quantified the extent of $\mathrm{HO}$ formation at the tenotomy site. Adipose-derived mesenchymal stem cells (ADMSCs) were harvested to evaluate osteogenic differentiation potential.
\end{abstract}

Results: Areas of developing $\mathrm{HO}$ demonstrated substantial enrichment of CD45+ leukocytes at 3-weeks postinjury. $\mathrm{HO}$ from Rag1 KO mice was substantially less mature with foci of cartilage and disorganized trabecular bone present 12 -weeks after injury. Rag1 $\mathrm{KO}$ mice formed $60 \%$ less bone compared to immunocompetent controls $(4.67 \mathrm{~mm} \pm 1.5 \mathrm{vs}$. $7.76 \mathrm{~mm} \pm .65 ; \mathrm{p}=.001)$. TRAP staining and immunofluorescent analysis of osteoprotegerin and pNF-kB demonstrated no appreciable difference in osteoclast number or activation. Alizarin red staining in vitro, however, demonstrated a significant decrease in osteogenic potential in immunocompromised mice compared to controls $(29.1 \mathrm{~mm} \pm .54$ vs. $12.1 \mathrm{~mm} \pm .14 ; p<0.001)$.

Conclusions: We demonstrate a prominent role for the adaptive immune system in the development of $\mathrm{HO}$. In the absence of mature $\mathrm{B}-/ \mathrm{T}$-lymphocytes, $\mathrm{HO}$ growth and development is attenuated. Furthermore, we demonstrate that mesenchymal populations from B/T-cell deficient mice are inherently less osteogenic. This study identifies a potential therapeutic role for modulation of the adaptive immune system in the treatment of $\mathrm{HO}$. 


\section{INTRODUCTION}

Heterotopic ossification $(\mathrm{HO})$ is the pathologic formation of ectopic bone within extra-skeletal structures commonly occurring as a result of musculoskeletal trauma, severe burns, neurologic injury, and orthopaedic intervention. ${ }^{1-5}$ With improved critical care, the incidence of $\mathrm{HO}$ is rising with improved survival after major trauma. ${ }^{2-5}$ Sequelae of post-traumatic $\mathrm{HO}$, including chronic pain, poor wound healing, and joint contracture, represent a significant barrier to functional recovery and independence. Treatment, including surgical excision, is limited and most patients never regain complete range of motion due to persistent or recurrent contractures. $^{3,6}$

Osteoimmunology or the role of the immune system in bony metabolism, is an area of growing interest in the study of $\mathrm{HO}$. Incidence and severity of $\mathrm{HO}$ after-trauma strongly correlates with the degree of injury and subsequent inflammation. ${ }^{1-4,7,8}$ Several inflammatory and/or infectious stimuli have been demonstrated to positively contribute to ectopic bone formation. ${ }^{8-12}$ Data from fracture healing, suggests a central role for the immunologic cytokine cascade in remodelling and repair after injury. ${ }^{13}$ Contributions from the innate or nonspecific immune system to $\mathrm{HO}$ have previously and repeatedly been demonstrated in trauma models. ${ }^{14-16}$ Further, in genetic models, such as fibrodysplasia ossificans progressive (FOP), histologic analysis of early lesions demonstrates the presence of a lymphocytic infiltrate suggesting a potential immunologic origin to even atraumatic ectopic bone pathologies. ${ }^{17}$

While it is likely that the immune system plays a role in $\mathrm{HO}$, the exact nature of this relationship is unclear. Early inflammatory markers IL-3, IL12, and IL-13 have been associated with $\mathrm{HO}$ development after trauma. ${ }^{18}$ The presence of $\mathrm{HO}$ also correlates with higher levels of IL-6, IL-10, MCP-1, IL-10, and MIP-1a. ${ }^{18}$ Further, the complement cascade has been implicated in the process of $\mathrm{HO} .{ }^{19}$ These cytokines likely regulate both osteoblastogenesis and osteoclastogenesis, suggesting a complex interplay between immunologic signals and bone formation.

Given the persistent nature of ectopic bone and its associated cytokine signals, the adaptive or acquired immune system is an attractive target to explore immunologic contributions to HO. Both B- and T-lymphocytes have demonstrated effects on bone formation in the regulation of mesenchymal stem cell (MSC) differentiation via IFN- $\gamma$ and TNF- $\alpha$, and inhibition of osteoclastogenesis through release of osteoprotegerin (OPG) ${ }^{20-23}$ In this study, we sought to identify whether the adaptive immune system and more specifically B- and T-lymphocytes, contribute to the formation and progression of ectopic bone after trauma. 


\section{METHODS AND MATERIALS}

Animals

Eight-week old male mice were used for all studies. Mice carrying a Rag1 knockout mutation (C57BL/Rag ${ }^{\text {T1Mom; }} \mathrm{n}=4$ ) preventing the maturation of both $\mathrm{B}$ - and T-lymphocytes were matched to C57BL/6 immunocompetent controls $(n=4)$. MicroCT and histologic analysis were performed at 12 -weeks after induction of $\mathrm{HO}$. A separate group of $\mathrm{C} 57 \mathrm{BL} / 6(\mathrm{n}=3)$ was raised for early histologic analysis of inflammatory populations present at the site of $\mathrm{HO}$ formation.

All animal procedures were carried out in accordance with the guidelines provided in the Guide for the Use and Care of Laboratory Animals from the Institute for Laboratory Animal Research (ILAR, 2011) and were approved by the Institutional Animal Care and Use Committee of the University of Michigan (PRO0001553).

\section{Burn/Tenotomy}

All mice received a partial thickness burn injury. Briefly, animals were anesthetized with 3-5\% inhaled isoflurane. Hair was clipped on the left dorsum to expose the skin. Partial-thickness burn was achieved by placing a metal brand heated to $60^{\circ} \mathrm{C}$ in a water bath against the exposed skin for 18 seconds. Each mouse received a concurrent Achilles tenotomy with aseptic, sharp dissection at the midpoint in the left hind limb. Pain management was achieved with subcutaneous injections of buprenorphine every 12 hours for 3 days.

\section{$\mu C T$ analysis}

In-vivo development of bone formation was assessed with longitudinal $\mu \mathrm{CT}$ scans at 12-weeks post injury ( $\mu \mathrm{CT}$ : Siemens Inveon, using $80 \mathrm{kVp}, 80 \mathrm{~mA}$ and $1100 \mathrm{~ms}$ exposure). Images were reconstructed and ectopic bone volume was calculated using total Hounsfield units to visualize the full extent of ectopic bone formation. All scans were also analyzed with threshold Hounsfield units (HU) of 800 (total), 1250 (intermediate-density), or 1800 (high-density) to determine the volume of tissues. Samples were then further analyzed to look at the arrangement of ectopic tissue in relation to the site of tenotomy. Abnormal bone adjacent to the calcaneus was defined as distal $\mathrm{HO}$ while ectopic tissue present in muscle or soft tissue areas and not immediately adjacent to native bone was defined as proximal $\mathrm{HO}$. Analysis of the scans was performed by a blinded investigator to minimize bias.

\section{Histologic Processing and Analyses}

At 1-, 3-, and 12-weeks post-operatively animals were euthanized for histology. ${ }^{24}$ Skin was removed from the tenotomy site and the hind limb was then fixed in buffered formalin solution for 24 hours at $4^{\circ} \mathrm{C}$.

Decalcification of the sample was completed with $19 \%$ ethylenediaminetetracetic acid (EDTA) solution for 2842 days at $4^{\circ} \mathrm{C}$. Decalcified tissues were dehydrated through graded ethanol, and paraffin embedded. Transverse sections were completed with a width of 5 microns, mounted on Superfrost plus slides (Fisher Scientific, Pittsburg, PA), and dried overnight at $37^{\circ} \mathrm{C}$. Histology including H\&E, Safranin O, and Aniline Blue stains were performed to visualize the anatomy and maturity of bone at the site. Tartrate-resistant acid phosphatase (TRAP) staining was performed to determine the role of osteoclasts in the evolution of the deposited $\mathrm{HO}$.

Immunofluorescent staining was performed as previously described for CD45 (553774; BD Pharmingen), SOX9 (sc-17341; Santa Cruz), Osteocalcin (sc-18319; Santa Cruz), Osteoprotegerin (AF459; RD Biosystems), Osterix (Bs-1110r; Bioss), Ki67 (Ab9260; Millipore), PDGFR-a (sc-338; Santa Cruz), and pNF-kB (sc-101752; Santa Cruz). We performed CD45 to visualize the presence of lymphocytes. To evaluate the maturity of the deposited HO, we stained for Sox9 to mark cartilage, Osterix to mark early bone formation and immature osteoblasts, and osteocalcin to mark the non-collagenous protein found within mature bone. PDGFR- $\alpha$ marks mesenchymal progenitor cell populations which are present at early time points of bone formation. Sections were deparaffinized and rehydrated in xylenes and graded ethanol. Antigen retrieval was performed with Citrate solution $\mathrm{pH}$ 6.0. Samples were then quenched for autofluorescence in $3 \%$ glycine before blocking and permeabilization. Primary antibodies were applied overnight at $4^{\circ} \mathrm{C}$. Appropriate dilutions were determined prior to achieving final images. After washing, fluorescently conjugated secondary antibodies tagged with either AlexaFluor 488 or AlexaFluor 594 (Life Technologies) were applied. Nuclear counterstain was achieved using Hoechst 33342 (Life Technologies) and mounted with ProLong Gold Antifade Reagent (Life Technologies). Appropriate primary antibody, secondary antibody, and autofluorescent controls were run simultaneously with each tested sample. 


\section{Microscopy}

All fluorescently stained images were taken using an Olympus BX-51 upright light microscope equipped with standard DAPI, 488, and TRITC reflector cubes attached to an Olympus DP-70 high-resolution digital camera. Images were visualized in Adobe Photoshop to perform vessel counts and to overlay cells for costaining.

Isolation and culture of primary adipose derived mesenchymal cells

Adipose-derived mesenchymal stem cells (ADMSCs) were harvested from the inguinal fat pads of mice ( $n=3$ per group) as previously described. ${ }^{25,26}$ Briefly, the inguinal fat pads were dissected, finely minced, and enzymatically digested in a $0.075 \%$ type A collagenase solution (Roche) and the MSCs were then isolated by adherent cell culture using standard procedures. Adherent ADMSCs were cultured and propagated in standard growth medium containing Dulbecco's Modified Eagle Medium (DMEM), 10\% fetal bovine serum, and 100 $\mathrm{IU} / \mathrm{ml}$ penicillin/streptomycin, and passaged on confluence by trypsinization.

\section{In vitro osteogenic differentiation}

MSCs were seeded onto six-well plates at a density of 100,000 cells/well and onto a 12-well plate at a density of 35,000 cells/well. After MSCs achieved $80 \%$ confluence, the cells were treated with osteogenic differentiation medium containing DMEM, $10 \%$ fetal bovine serum, $100 \mathrm{~g} / \mathrm{ml}$ ascorbic acid, $10 \mathrm{mM}$ Betaglycerophosphate, and $100 \mathrm{IU} / \mathrm{ml}$ penicillin/streptomycin. Early osteogenic differentiation was assessed by alkaline phosphatase (ALP) stain after 7 days in osteogenic differentiation medium (ODM). Alizarin red staining for bone mineral deposition and photometric quantification was completed at 2 weeks.

\section{Statistical analysis}

Data were analyzed using SPSS software (IBM). Means and standard deviations were calculated from numerical data and statistical analysis was performed using Student's T-Test. In figures, bar graphs represent means, whereas error bars represent one standard deviation. For all assays, significance was defined as a $p<$ 0.05 . 


\section{RESULTS}

\section{Lymphocytic cells are enriched at and persist within sites of developing HO after trauma.}

Initially we sought to identify the course of leukocyte enrichment at the site of injury. Leukocytes positive for CD45+ were significantly upregulated locally within one week of tenotomy compared to uninjured controls. Leukocytes remained highly enriched at 3-weeks post-injury demonstrating the persistence of the immunologic response even at this time point (Fig. 1A). We then analyzed the composition of inflammatory cells at the site of developing $\mathrm{HO}$. Unsurprisingly cells of the innate immune system composed the majority of the inflammatory infiltrate at 1-week (Fig. 1B). By 3-weeks, however, lymphocytes, were found in high frequency (Fig. 1B).

\section{Combined B-/T-cell deficiency decreases both volume and maturity of ectopic bone after trauma.}

We then utilized Rag1 KO mice, a population previously demonstrated to have a complete absence of mature B- and T-cells, to study the contribution of the adaptive immune system to HO formation. Twelve weeks after burn/tenotomy, both Rag1 and immunocompetent controls were sacrificed for radiographic and histologic analysis. On histologic analysis, ectopic bone formation in Rag1 immunocompromised mice was substantially less as a result of diminished chondrogenic differentiation compared to the control mice. Even at twelve weeks, there were immature trabeculae present within the $\mathrm{HO}$ of the immunocompromised mice, while the control mice had mature, well-ossified bone present at the site. This suggests that $B$ and $T$ cells may play a role in progression of endochondral ossification as it relates to $\mathrm{HO}$ formation (Fig. 2A,B). This was confirmed with immunofluorescent staining for SOX9 and Osteocalcin (OCN) to examine the presence of chondrocytes and mature osteoblasts in the developing HO. Rag1 mice retained SOX9 staining at 12-weeks while, immunocompetent controls demonstrated enrichment of $\mathrm{OCN}+$ cells. This demonstrates that the bone of the immunocompromised mice was substantially less mature than that of the control mice (Fig. 2C,D). Immunophenotypic characterization of cells of the inflammatory anlagen revealed no difference in cellular proliferation between mutant and control mice, as demonstrated by Ki-67 immunostaining (Fig. 3A). Cells within the developing anlagen of Rag1KO mice revealed increased signaling of platelet derived growth factor receptor-alpha (PDGFR- $\alpha$ ) and Osterix (Osx), indicating the relative immaturity of the mutant anlagen when compared to controls (Fig. 3B).

Furthermore, on MicroCT analysis, the total volume of $\mathrm{HO}$ in Rag1 mice was significantly lower than in immunocompetent controls (Fig. 4A). Rag1 mice formed $60 \%$ less bone compared to the immunocompetent mice $\left(4.67 \pm 1.5 \mathrm{~mm}^{3}\right.$ vs. $7.76 \pm .65 \mathrm{~mm}^{3} \mathrm{p}=.001$; Fig. 4B). Interestingly, while immunocompetent mice developed $\mathrm{HO}$ at both the proximal and distal end of the transected tendon, Rag1KO mice had no proximal bone formation (Fig. 4A).

\section{B-/T-cell deficient mice do not demonstrate deficiencies in osteoclastogenesis at sites of $\mathrm{HO}$.}

To examine the impact of combined B-/T-lymphocyte deficiency on bone maturation, we examined differences in signaling and osteoclast function at the site of $\mathrm{HO}$ (Fig. 5A). As B- and T-cells are known to regulate osteoclastogenesis via production of OPG and attenuation of pNF-kB signals we wanted to identify whether Rag1 KO mice might demonstrate a difference in signaling at the site of HO. Immunofluorescent staining for OPG and pNF-kB demonstrated no difference at 12-weeks (Fig. 5B). Additionally, there were no differences in osteoclast function as demonstrated using TRAP stain. (Fig. 5C). Therefore, the difference in bone maturation between immunocompromised mice and controls is not a reflection of differences in osteoclast function or signaling.

\section{MSCs from B-/T-cell deficient mice demonstrates decreased osteogenic capacity.}

Given that osteoclasts in $\mathrm{HO}$ were not appreciably different between the two groups, we decided to examine the effect of B-/T-cell deficiency on mesenchymal populations. By alizarin red staining, we found that adipose-derived MSCs (ADMSCs) from Rag1 mice were significantly less osteogenic than ADMSCs from immunocompetent controls (29.1 \pm .54 vs. $12.1 \pm .14 ; p<0.001$; Fig. 5D). Thus, the difference in bone composition between the control and immunocompromised mice is most likely a result of $B$ and $T$ cell modulation of the osteogenic potential inherent to the local mesenchymal population. 


\section{DISCUSSION}

While previous studies have mainly focused on the contribution of the innate immune system to heterotopic ossification, we have demonstrated that lymphocytic cell populations are also critical to the process of $\mathrm{HO}$ formation. In this study, we first demonstrate that a significant inflammatory CD45+ cell population migrates to the site of trauma, and later contributes to the process of HO. B- and T-cell deficient mice form less heterotopic bone at the site of trauma compared to immunocompetent mice, despite no alteration in osteoclastogenesis in the former group. Histology corroborates reduced and delayed ossification of the cartilage anlage in immunocompromised mice, indicating a possible step at which B- and T-cells likely exert their function. The presence of PDGFR- $\alpha$, a characteristic marker of mesenchymal progenitor cells, and Osx, a transcription factor that regulates osteoblast differentiation and marks osteogenic precursors, the cellular population in Rag1KO mice is consistent with immature stages of $\mathrm{HO}$ development. ${ }^{27}$ The absence of a difference in proliferation, as visualized by $\mathrm{Ki}-67$ immunostaining between Rag1KO and control mice also suggests that the delayed ossification cannot be attributed to differences in cellular proliferation. Taken together, the absence of adaptive immune cells impacts the maturation of the cells within the traumatic anlage of Rag1 KO mice. Similarly, in vitro culture of mesenchymal stem cells demonstrates reduced osteogenic potential of lymphocyte-depleted mice. Based on this data, we demonstrate that the adaptive immune system plays a prominent role in the inflammatory cascade leading to ectopic bone formation.

Our results are consistent with previous studies that elucidate the complex role of B- and T-cells in physiologic bone homeostasis. ${ }^{21-23}$ Interestingly, populations of T-cells heterogeneously modulate osteogenesis. While CD8+ T-cells block bone formation, regulatory T-cells are thought to enhance osteogenesis by decreasing levels of pro-inflammatory cytokines, IFN- $\gamma$ and TNF- $\alpha$, resulting in improved healing within a bony defect. ${ }^{20}$ Similarly, B-cells are known to affect bone synthesis and remodeling. B-cells enhance bone formation through the release of osteoprotegerin, a soluble ligand that binds to RANKL, preventing stimulation of the RANK receptor complex responsible for osteoclast activation. ${ }^{24} \mathrm{~B}$-cells also mediate apoptosis of osteoclasts through the release of TGF $\beta$. Although paracrine contributions to bone homeostasis from each cell type have been documented, the coordinated interplay between these two cell populations also proves critical. The stimulation of the B-cell CD40+ receptor by T-cells stimulates osteoprotegerin production, and both B and T cells are involved in basal bone turnover and regulation. ${ }^{24}$ Interestingly, the absence of lymphocytes did not significantly increase osteoclast activity on histological analysis, suggesting an alternative pathway of mediating reduced osteogenic potential. Despite established contributions of the adaptive immune system to osteogenesis in the setting of normal bone formation, these cell populations potentially modulate the formation of pathologic bone through alternative mechanisms in the setting of trauma.

There are important limitations of this study to consider. Although we demonstrate the importance of Band T-cells in the process of $\mathrm{HO}$, we do not isolate specific factors secreted by each cell type. Additional studies are required to confirm whether the presence or absence of B- and T-cells independently are necessary to impact $\mathrm{HO}$ formation, and how these cells uniquely interact with the resident cell populations at the site of injury. Importantly, however, we demonstrate that a potential target for pharmacologic intervention lies within modulation of the adaptive immune system. Given the numerous interactions between various components of the adaptive immune system and osteogenesis, it is important to define these relationships to optimize our use of currently available pharmacologic agents as potential applications to the management of $\mathrm{HO}$. 


\section{CONCLUSION}

We demonstrate the impact of the adaptive immune system on the pathogenesis of heterotopic ossification. The depletion of a lymphocytic population yields reduced and delayed ossification of soft tissue in the setting of trauma. In the absence of B- and T-cells, the attenuation of this osteogenic response is not conferred by modulation of osteoclastogenesis, but rather by an inherent limitation of osteogenic potential. This study identifies the significance a potential therapeutic role for modulation of the adaptive immune system in the treatment of heterotopic ossification and suggests that further exploration of the relationship between the immune system and diseases of ectopic bone formation may prove useful. 


\section{FIGURE LEGEND}

Figure 1. Areas of developing HO demonstrate enrichment and persistence of lymphocytes. A. CD45 stain (green) demonstrating enrichment of leukocytes at the site of tenotomy by 1 -week persisting into the development of early $\mathrm{HO}$ at 3-weeks. B. H\&E at 10 and $60 x$ demonstrating the presence of lymphocytes in and adjacent to areas of developing $\mathrm{HO}$ by 1 -week after tenotomy. Red box identifies area of 60x magnification.

Figure 2. Ectopic bone in Rag1 $\mathrm{KO}$ mice is less mature than in immunocompetent controls $\mathrm{A}$. $\mathrm{H} \& \mathrm{E}$ staining of Rag1 $\mathrm{KO}$ and wild-type $\mathrm{HO}$ at 12-weeks demonstrating increased ossification and trabecular maturation in the immunocompetent mice. B. Safranin O stain (red) with Fast Green FCF counterstain (blue/green) demonstrating the presence of cartilage in Rag1 $\mathrm{KO}$ mice suggesting incomplete endochondral ossification. C. SOX9 immunostaining (red) demonstrating the presence of early chondrocytes in $\mathrm{HO}$ from Rag1 KO but not wild-type mice. D. OCN immunostaining (red) demonstrating enrichment of mature osteoblasts in wild-type mice compared to Rag1 KO. All stains at 4x and 20x magnification. Black or white boxes demonstrate area of $20 x$ magnification.

Figure 3. Injury site of Rag1KO mice defined by immunophenotypic markers of delayed differentiation. A. Immunostaining of proliferation marker Ki-67 demonstrates no difference between proliferation in the mutant and control mice. Images taken at 20x magnification. B. PDGFR- $\alpha$ and OSX staining demonstrating signal positivity in anlagen of Rag1 $\mathrm{KO}$ and not control mice, suggesting mesenchymal cell population at earlier stage of $\mathrm{HO}$ development. Images taken at 40x magnification.

Figure 4. Decreased ectopic bone formation in Rag1 KO mice. A. MicroCT reconstructions with accompanying aniline blue stained histologic cross-section of $\mathrm{HO}$ at 12-weeks post-injury demonstrating the presence of ectopic bone both proximal and distal to the transected tendon in wild-type controls. Proximal HO is absent in the Rag1 KO mice. Yellow dotted lines highlight areas of proximal HO. Red dotted lines highlight areas of distal HO. B. Quantification of total $\mathrm{HO}$ volumes in the distal hind limb. All error bars represent standard deviation. Black asterisks on graph represent $p<0.05$.

Figure 5. Osteogenic differentiation, but not osteoclastogenesis is diminished in Rag1 KO mice. A. Schematic for potential ways that B- and/or T-cell deficiency may affect ectopic bone formation. B. pNF-KB (green) and OPG (red) immunostaining demonstrating the presence of pro- and anti-osteoclastogenic signals at the site of $\mathrm{HO}$ 12-weeks after injury demonstrating similar patterns in both groups. C. TRAP staining demonstrating comparable presence of osteoclasts in $\mathrm{HO}$ from Rag1 KO and wild-type mice. Red arrows highlight areas of positive TRAP staining. D. Alizarin red staining of ADMSCs from wild-type and Rag1 KO mice demonstrating decreased osteogenic differentiation in immunodeficient mice. 


\section{REFERENCES}

1. Orchard GR, Paratz JD, Blot S, Roberts JA. (2015). Risk Factors in Hospitalized Patients With Burn Injuries for Developing Heterotopic Ossification--A Retrospective Analysis. J Burn Care Res. 2015 JulAug;36(4):465-70. doi: 10.1097/BCR.0000000000000123.

2. Pavey GJ, Polfer EM, Nappo KE, Tintle SM, Forsberg JA, Potter BK. (2015). What Risk Factors Predict Recurrence of Heterotopic Ossification After Excision in Combat-related Amputations? Clin Orthop Relat Res. 2015 Sep;473(9):2814-24. doi: 10.1007/s11999-015-4266-1.

3. Garland DE. A clinical perspective on common forms of acquired heterotopic ossification. Clinical orthopaedics and related research 1991:13-29.

4. Forsberg JA, Pepek JM, Wagner S, et al. Heterotopic ossification in high-energy wartime extremity injuries: prevalence and risk factors. The Journal of bone and joint surgery American volume 2009;91:1084-91.

5. Forsberg JA, Potter BK. Heterotopic ossification in wartime wounds. Journal of surgical orthopaedic advances 2010;19:54-61.

6. Sakellariou VI, Grigoriou E, Mavrogenis AF, Soucacos PN, Papagelopoulos PJ. (2012). Heterotopic ossification following traumatic brain injury and spinal cord injury: insight into the etiology and pathophysiology. J Musculoskelet Neuronal Interact. 2012 Dec;12(4):230-40.

7. Ranganathan K, Loder S, Agarwal S, Wong VC, Forsberg J, Davis TA, Wang S, James AW, Levi B. (2015). Heterotopic Ossification: Basic-Science Principles and Clinical Correlates. J Bone Joint Surg Am. 2015 Jul 1;97(13):1101-11. doi: 10.2106/JBJS.N.01056.

8. Nelson ER, Wong VW, Krebsbach PH, Wang SC, Levi B. (2012). Heterotopic ossification following burn injury: the role of stem cells. J Burn Care Res. 2012 Jul-Aug;33(4):463-70. doi:

10.1097/BCR.0b013e31825af547.

9. Pavey GJ, Qureshi AT, Hope DN, Pavlicek RL, Potter BK, Forsberg JA, Davis TA. (2015). Bioburden Increases Heterotopic Ossification Formation in an Established Rat Model. Clin Orthop Relat Res. 2015 Sep;473(9):2840-7. doi: 10.1007/s11999-015-4272-3.

10. Brown KV, Dharm-Datta S, Potter BK, Etherington J, Mistlin A, Hsu JR, Clasper JC. (2010). Comparison of development of heterotopic ossification in injured US and UK Armed Services personnel with combatrelated amputations: preliminary findings and hypotheses regarding causality. J Trauma Inj Infect Crit Care. 2010;69:S116-S122

11. Thilak J, Panakkal JJ, Kim TY, Goodman SM, Lee SS, Salvati EA. (2015). Risk Factors of Heterotopic Ossification Following Total Hip Arthroplasty in Patients With Ankylosing Spondylitis. J Arthroplasty. 2015 Jun 14. pii: S0883-5403(15)00525-2. doi: 10.1016/j.arth.2015.06.013. [Epub ahead of print]

12. Salisbury E, Rodenberg E, Sonnet C, Hipp J, Gannon FH, Vadakkan TJ, Dickinson ME, Olmsted-Davis EA, Davis AR. (2011). Sensory nerve induced inflammation contributes to heterotopic ossification. J Cell Biochem. 2011 Oct;112(10):2748-58. doi: 10.1002/jcb.23225.

13. Toben D, Schroeder I, El Khassawna T, Mehta M, Hoffmann JE, Frisch JT, Schell H, Lienau J, Serra A, Radbruch A, Duda GN. (2011). Fracture healing is accelerated in the absence of the adaptive immune system. J Bone Miner Res. 2011 Jan;26(1):113-24. doi: 10.1002/jbmr.185.

14. Genêt F, Kulina I, Vaquette C, Torossian F, Millard S, Pettit AR, Sims NA, Anginot A, Guerton B, Winkler IG, Barbier V, Lataillade JJ, Le Bousse-Kerdilès MC, Hutmacher DW, Levesque JP. (2015).

Neurological heterotopic ossification following spinal cord injury is triggered by macrophage-mediated inflammation in muscle. J Pathol. 2015 Jun;236(2):229-40. doi: 10.1002/path.4519. Epub 2015 Mar 26.

15. Salisbury E, Sonnet C, Heggeness M, Davis AR, Olmsted-Davis E. (2010). Heterotopic ossification has some nerve. Crit Rev Eukaryot Gene Expr. 2010;20(4):313-24.

16. Kan L, Mutso AA, McGuire TL, Apkarian AV, Kessler JA. (2014). Opioid signaling in mast cells regulates injury responses associated with heterotopic ossification. Inflamm Res. 2014 Mar;63(3):207-15. doi: 10.1007/s00011-013-0690-4. Epub 2013 Dec 11.

17. Gannon FH, Valentine BA, Shore EM, Zasloff MA, Kaplan FS. (1998). Acute lymphocytic infiltration in an extremely early lesion of fibrodysplasia ossificans progressiva. Clin Orthop Relat Res. 1998 Jan;(346):19-25.

18. Forsberg JA, Potter BK, Polfer EM, Safford SD, Elster EA. (2014). Do inflammatory markers portend heterotopic ossification and wound failure in combat wounds? Clin Orthop Relat Res. 2014 Sep;472(9):2845-54. doi: 10.1007/s11999-014-3694-7. Epub 2014 May 31. 
19. Evans KN1, Forsberg JA, Potter BK, Hawksworth JS, Brown TS, Andersen R, Dunne JR, Tadaki D, Elster EA. (2012). Inflammatory cytokine and chemokine expression is associated with heterotopic ossification in high-energy penetrating war injuries. J Orthop Trauma. 2012 Nov;26(11):e204-13. doi: 10.1097/BOT.0b013e31825d60a5.

20. $\quad$ Liu Y, Wang L, Kikuiri T, Akiyama K, Chen C, Xu X, Yang R, Chen W, Wang S, Shi S. (2011). Mesenchymal stem cell-based tissue regeneration is governed by recipient $\mathrm{T}$ lymphocytes via IFN- $\mathrm{Y}$ and TNF- $\alpha$. Nat Med. 2011 Nov 20;17(12):1594-601. doi: 10.1038/nm.2542.

21. Li S, Li T, Chen Y, Nie Y, Li C, Liu L, Li Q, Qiu L. (2015). Granulocyte Colony-Stimulating Factor Induces Osteoblast Inhibition by B Lymphocytes and Osteoclast Activation by T Lymphocytes during Hematopoietic Stem/Progenitor Cell Mobilization. Biol Blood Marrow Transplant. 2015 Aug;21(8):138491. doi: 10.1016/j.bbmt.2015.05.005. Epub 2015 May 16.

22. Könnecke I, Serra A, El Khassawna T, Schlundt C, Schell H, Hauser A, Ellinghaus A, Volk HD, Radbruch A, Duda GN, Schmidt-Bleek K. (2014). T and B cells participate in bone repair by infiltrating the fracture callus in a two-wave fashion. Bone. 2014 Jul;64:155-65. doi: 10.1016/j.bone.2014.03.052. Epub 2014 Apr 8.

23. Leibbrandt A, Penninger JM. (2008). RANK/RANKL: regulators of immune responses and bone physiology. Ann N Y Acad Sci. 2008 Nov;1143:123-50. doi: 10.1196/annals.1443.016.

24. Peterson, Jonathan R., Sara De La Rosa, Oluwatobi Eboda, Katherine E. Cilwa, Shailesh Agarwal, Steven R. Buchman, Paul S. Cederna et al. "Treatment of heterotopic ossification through remote ATP hydrolysis." Science translational medicine 6, no. 255 (2014): 255ra132-255ra132.

25. Peterson, Jonathan R., Oluwatobi Eboda, Shailesh Agarwal, Kavitha Ranganathan, Steven R. Buchman, Min Lee, Stewart C. Wang, Yuji Mishina, and Benjamin Levi. "Targeting of ALK2, a Receptor for Bone Morphogenetic Proteins, Using the Cre/lox System to Enhance Osseous Regeneration by Adipose-Derived Stem Cells." Stem cells translational medicine 3, no. 11 (2014): 1375.

26. Loder, Shawn, Jonathan R. Peterson, Shailesh Agarwal, Oluwatobi Eboda, Cameron Brownley, Sara DeLaRosa, Kavitha Ranganathan, Paul Cederna, Stewart C. Wang, and Benjamin Levi. "Wound healing after thermal injury is improved by fat and adipose-derived stem cell isografts." Journal of burn care \& research: official publication of the American Burn Association 36, no. 1 (2014): 70-76.

27. Mori H, Kitazawa R, Mizuki S, Nose M, Maeda S, Kitazawa S. (2002). RANK ligand, RANK, and OPG expression in type II collagen-induced arthritis mouse. Histochem Cell Biol. 2002 Mar;117(3):283-92. Epub 2002 Jan 26. 


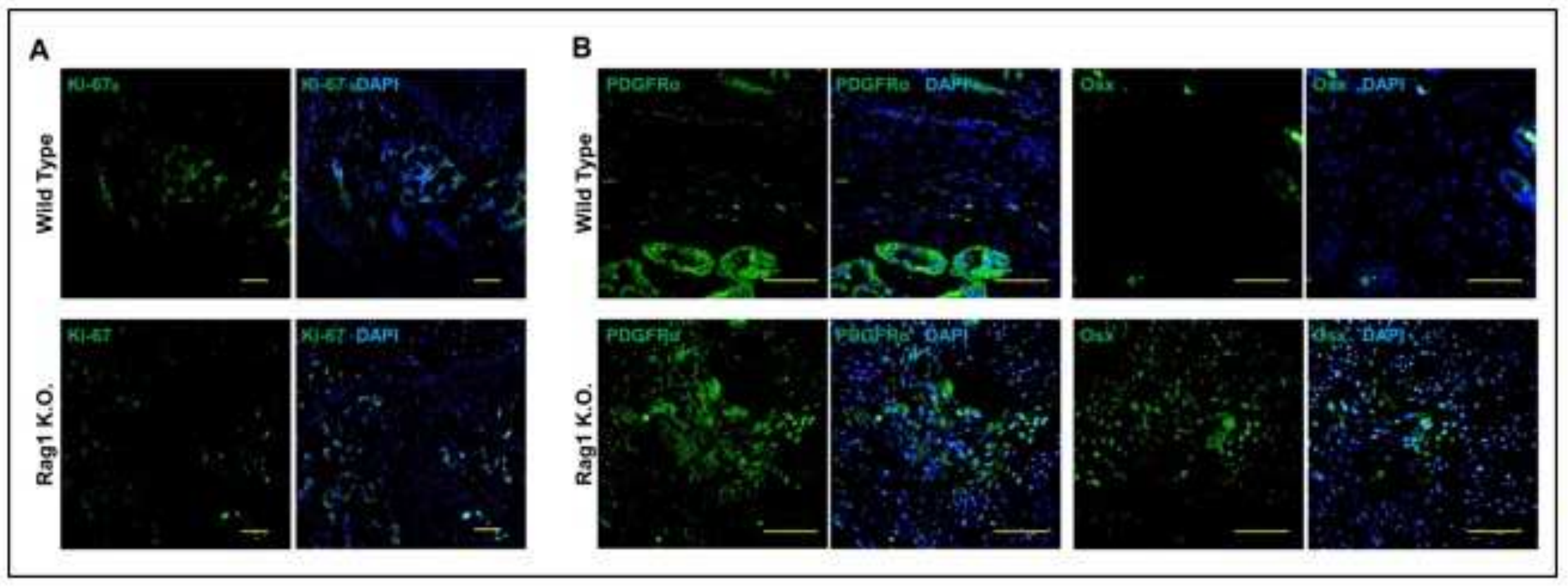

B
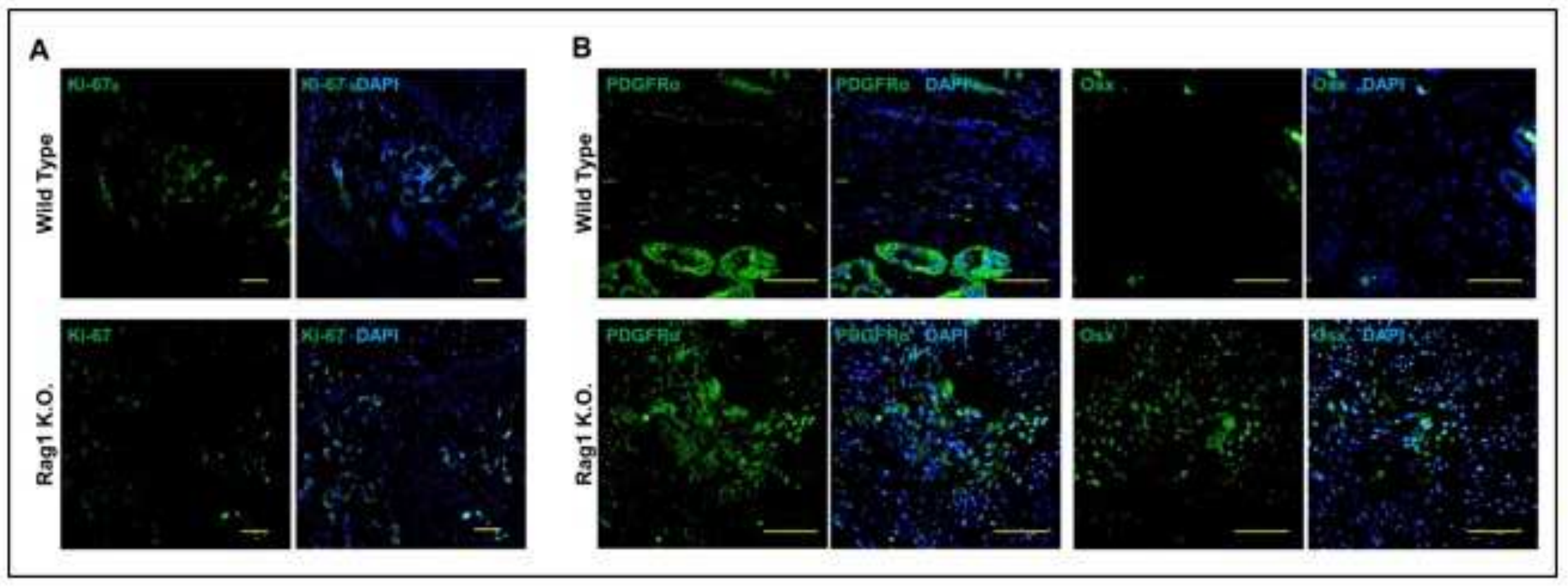


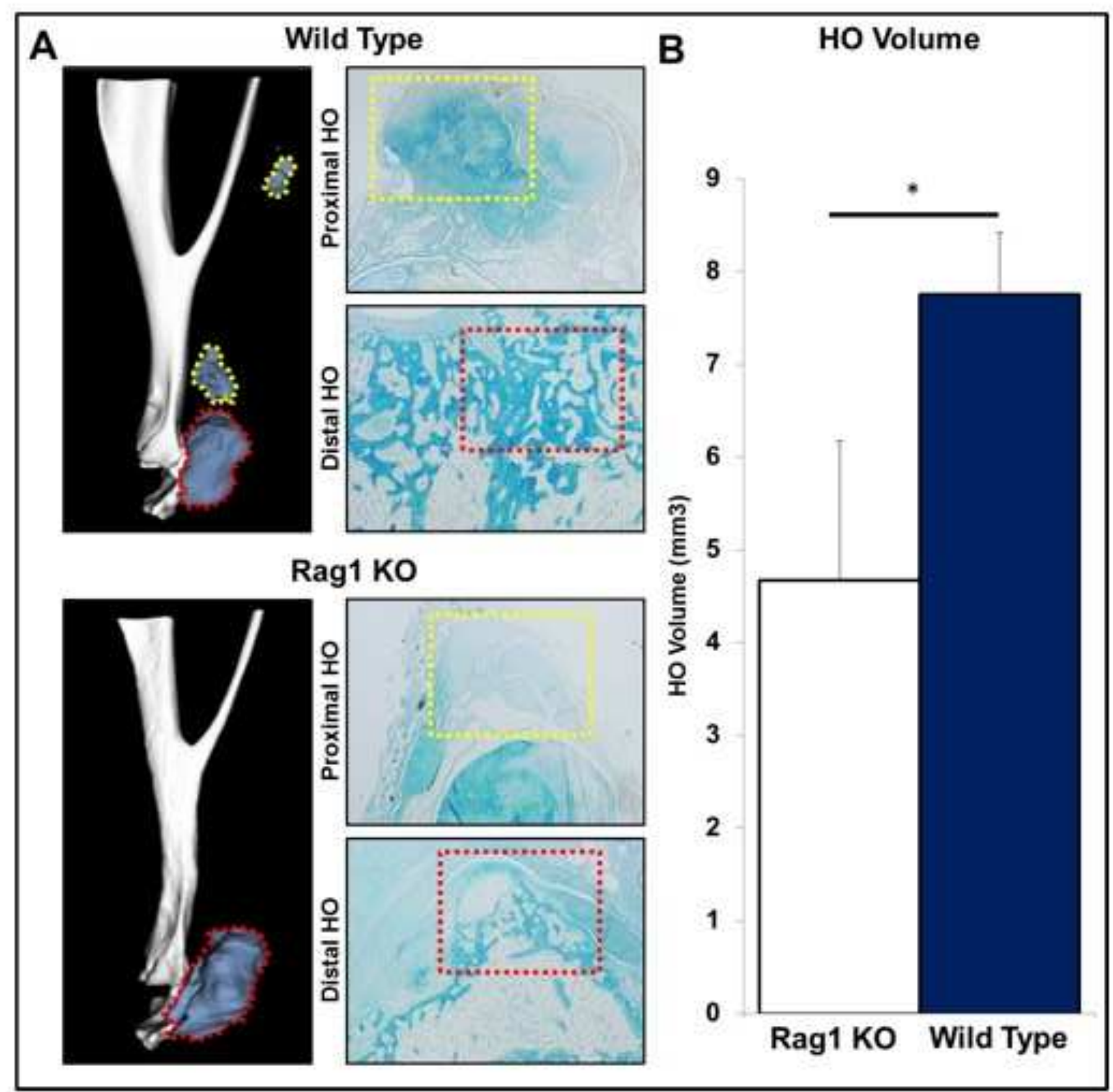




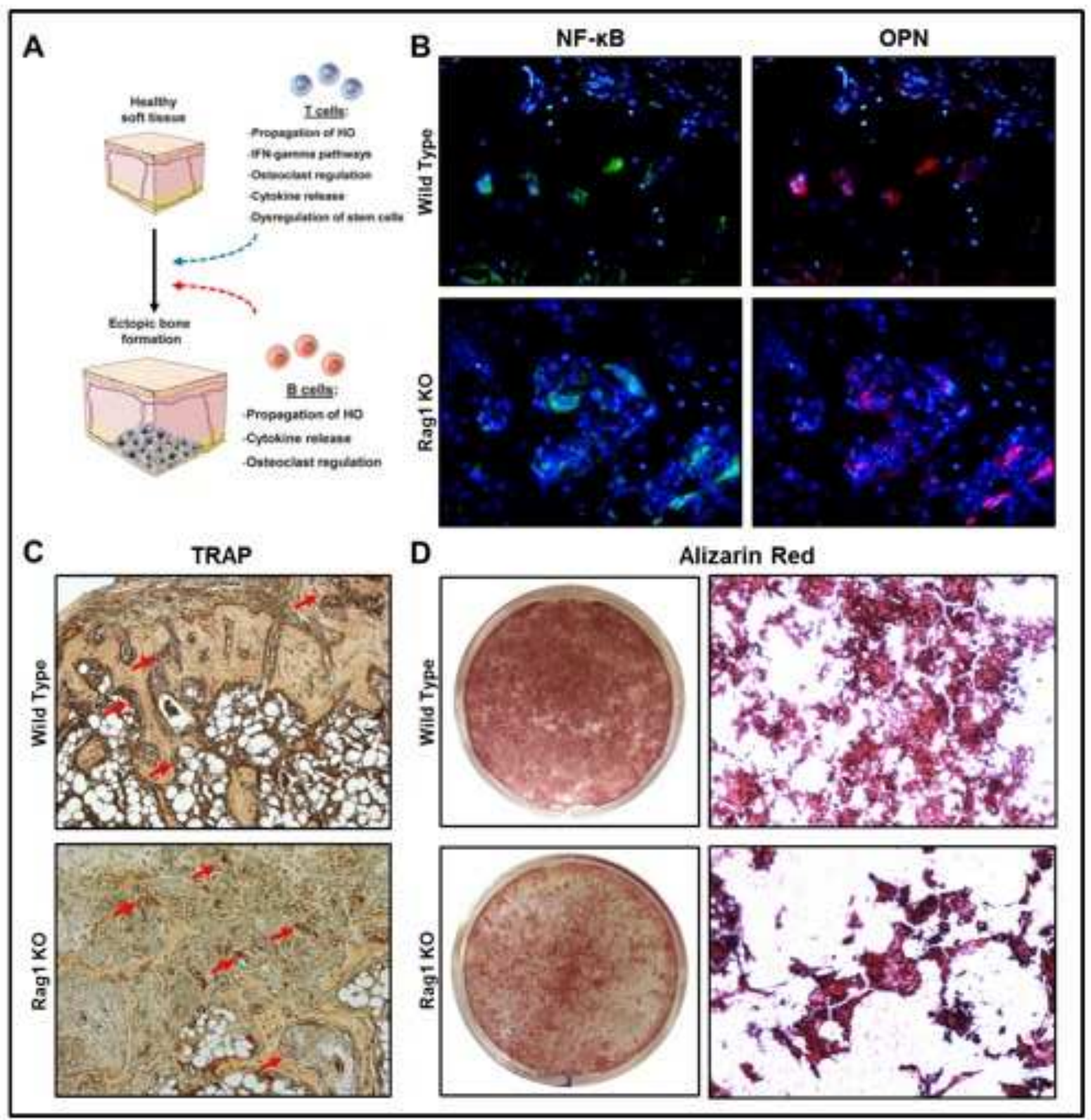

